\title{
Fractionally Spaced Constant Modulus Equalizer with Recognition Capability for Digital Array Radar
}

\author{
Feng Wang, Shuang Wei, and Defu Jiang \\ Array and Information Processing Laboratory, College of Computer and Information, Hohai University, No. 8, \\ West Focheng Road, Jiangning District, Nanjing 211100, China
}

Correspondence should be addressed to Feng Wang; jihonghope@aliyun.com

Received 18 September 2016; Revised 19 January 2017; Accepted 30 January 2017; Published 21 February 2017

Academic Editor: Haranath Kar

Copyright (C) 2017 Feng Wang et al. This is an open access article distributed under the Creative Commons Attribution License, which permits unrestricted use, distribution, and reproduction in any medium, provided the original work is properly cited.

Fractionally spaced blind equalizer (BE) based on constant modulus criteria is exploited to compensate for the channel-to-channel mismatch in a digital array radar. We apply the technique of recognition to improve the stability and reliability of the BE. The surveillance of the calibration signal and the convergence property of $\mathrm{BE}$ are both implemented with recognition description words. $\mathrm{BE}$ with cognitive capability is appropriate for the equalization of a digital array radar with thousands of channels and hundreds of working frequencies, where reliability becomes the most concerned indicator. The improvement of performance in the accidental scenarios is tested via numerical simulations with the cost of increased computational complexity.

\section{Introduction}

Compared with the analog active phased array radar, a digital array radar (DAR) or a radar with digital beamforming architecture has the potential of increasing system dynamic range in the main beam, more freedoms for adaptive-nulling, more effective time-energy management, and so on [1]. An active DAR hosts thousands of digital transmit/receive modules, in which low noise amplification, frequency mixing, and band pass filtering are performed as the analog receiving channel. The variability in amplitude and phase in the analog receiving channel leads to the channel-to-channel mismatch, which is the main reason of high side lobe levels for digital beam forming (DBF) and low jamming suppression ratio when using side lobe cancellation [2]. Thereby, the calibration system is essential for a DAR to achieve high performance, though it is quite expensive in time and operative costs [3].

Calibration system with single frequency injections is usually used in a DAR, compensating the channel-to-channel difference with one tap weight for each working frequency bin. It is only a compensation of one frequency point, so that the differences of amplitudes and phases in other frequencies in the instantaneous frequency bandwidth remain even for a narrow band system [4].
Considering the channel-to-channel differences of the multiple frequencies in the bandwidth, adaptive equalization with a finite impulse response structure is employed in each of the channels to correct the channel transfer functions in [2]. All the channels match the reference channel in gain and phase by injecting a linear frequency modulation calibration signal sweeping the instant bandwidth. The data used for calculating the weights of the equalizers must be transferred to a calculating unit down the array panel in such a calibration system. The equalizer weight after calculation should again be transferred back to the digital transmit/receive modules via an uplink channel. Debugging and testing of such a transferring system is too complicated for a DAR with thousands of channels.

To simplify the implementation of the calibration system and to improve the performance of a DAR simultaneously, an internal calibration technique based on blind equalization (BE) [5] is proposed in this paper. BE is an approach commonly utilized to counter the effects of intersymbol interferences in communication systems. The difference between $\mathrm{BE}$ and adaptive equalization lies in that $\mathrm{BE}$ needs no training sequence, which means more efficient use of the bandwidth in the communication scenarios [6]. However, for a radar calibration system, calibration signal is a known sequence 
to the system designer. The significance of the convergence without training sequence lies in that we can capture the set of calibration signal randomly, without considering the accuracy of the timing signals. Another merit of BE is that the closed-loop of such a calibration process is only implemented within each individual digital transmit/receive modules, without linking with other subsystems.

Of all the blind equalization algorithms, constant modulus algorithm (CMA) is the most classical and implemented in practice $[5,6]$. CMA is a stochastic gradient based algorithm exploiting fractionally spaced structure or symbol spaced structure. Fractionally spaced equalizer (FSE) generally performs better than symbol spaced equalizer due to its improved time phase selectivity and global convergence $[7,8]$. Hence, FSE relaxes the demand on the accuracy of the timing signal, which is significant for a DAR requiring a synchronization of thousands of channels. Radar systems exploiting FSEs should set their sampling data rate much higher than the instantaneous band width. Since the input data of the FSEs is above the Nyquist rate, the computational burden increases with the increase of the dimension of the FSE subchannels.

Incorrect equalizer coefficients will lead to serious performance degradation for digital beamforming and the subsequent processing. For a DAR with thousands of channels and hundreds of working frequencies, the implementation of the fractionally spaced CMA (FS-CMA) reaches millions of times in one day. The reliability of achieving a correct equalizer is of great significance in such a complicated system. The reason lies in that the error in one channel in digital domain will be large enough to cover the weak target signal of the radar. To improve the reliability of BE, we induce the concept of recognition [9], which consists of the recognition of the calibration signal and the convergence status of the FS-CMA. As well as the mean square error (MSE), the convergence property of the equalizer and the quality of the calibration signal are all under the surveillance of the recognition approach. The recognitive capability exploited for channel equalization is part of the concept of a recognitive radar, which has been widely discussed [10-12]. The recognition of the calibration signal is also a concept of knowledge based radar [13, 14], since the signal is a priori knowledge.

In this paper, Section 2 describes the conventional multichannel model of FS-CMA. Section 3 describes our FSCMA with recognition function in detail and illustrates the approach of constructing the recognition description word (RDW). We validate the performance of the proposed approach in Section 4 through computer simulations. Conclusions are given in Section 5.

\section{The Model of the Conventional FS-CMA [6]}

The equivalent multichannel model of the conventional FSCMA is shown in Figure 1.

We define the time interval of the Nyquist sampling frequency as $T$. Suppose that we sample the analog signal with a data rate of $M$ times the Nyquist sampling rate, with signal samples spaced $T / M$ apart. The output of the $i$ th subchannel becomes [15]

$$
x_{i}(k)=\mathbf{c}_{i} \otimes p(k)+\xi_{i}(k),
$$

where $k$ is the discrete time instant, $x_{i}(k)$ is the output data of the $i$ th equivalent subchannel, $p(k)$ is the common transmitted signal in stochastic phase-shift keying modulation, $\mathbf{c}_{i}=\left[c_{i}^{0}, c_{i}^{1}, c_{i}^{2}, \ldots, c_{i}^{\mathrm{Q}-1}\right]^{T}$ is the discrete channel impulse response of the $i$ th subchannel with $Q$ referring to the number of coefficients, $\xi_{i}(k)$ is the additive white Gaussian noise in the $i$ th subchannel, and $\otimes$ is the operator of convolution.

The output of the $i$ th subequalizer can be written as

$$
y_{i}(k)=\mathbf{f}_{i}(k) \otimes x_{i}(k)
$$

where $\mathbf{f}_{i}(k)$ denotes the $i$ th subequalizer of $N_{f} \times 1$ dimension. The output of the fractionally spaced equalizer is a combination of all the subequalizers

$$
z(k)=\frac{1}{M} \sum_{i=0}^{M-1} y_{i}(k) .
$$

The cost function of CMA in accordance with the minimum mean square error (MMSE) criterion can be expressed as

$$
\chi=\mathrm{E}\left\{\left(\gamma-|z(k)|^{2}\right)\right\}
$$

where $\mathrm{E}\{\cdot\}$ represents mathematical expectation, $|\cdot|$ denotes the modulus calculation, and [6]

$$
\gamma=\frac{\mathrm{E}\left\{|p(k)|^{4}\right\}}{\mathrm{E}\left\{|p(k)|^{2}\right\}} .
$$

Parameter $\gamma$ in (5) represents the constant modulus of the calibration signal. As the modulus of the equalizer output varies above or below $\gamma$, we get an error term to determine the update direction of the equalizer, which is described as follows.

The error term for equalizer update obtained by nonlinear transform (NLT) is of the following form:

$$
e(k)=z(k)\left[\gamma-|z(k)|^{2}\right] .
$$

The input regressor of the $i$ th equalizer can be defined as

$$
\mathbf{X}_{i}(k)=\left[\begin{array}{llll}
x_{i}(k) & x_{i}(k-1) & \cdots & x_{i}\left(k-N_{f}+1\right)
\end{array}\right]^{T} ;
$$

thereby we can express the iteration process of the $i$ th equalizer as

$$
\mathbf{f}_{i}(k+1)=\mathbf{f}_{i}(k)+\mu_{1} \cdot \mathbf{X}_{i}^{*}(k) e(k),
$$

where $\mu_{1}$ denotes the step size factor and $[\cdot]^{\bullet}$ denotes the conjugate operation. The convergence speed and residual mean square error of the FS-CMA equalizer are controlled by $\mu_{1}$; that is, larger $\mu_{1}$ means fast convergence rate and lager residual mean square error while less $\mu_{1}$ means slow convergence rate and less residual mean square error. 


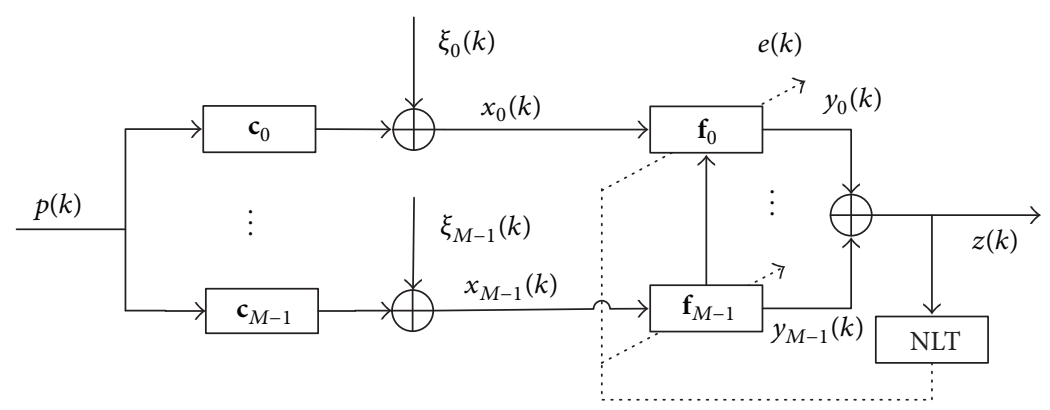

FIgURE 1: Multichannel model of conventional FS-CMA.

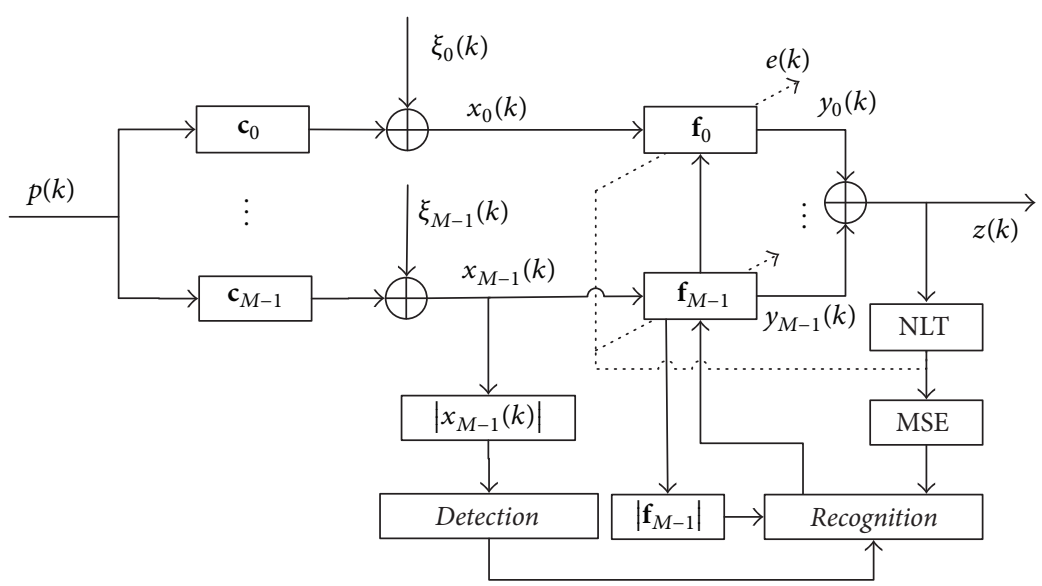

FIgURE 2: Multichannel model of FS-CMA with recognition capability.

\section{FS-CMA with Recognition Capability}

The $\mathrm{BE}$ in a digital transmit/receive module runs in an automatic mode, and the validity of the results relies on two basic conditions. One is the correctness of the calibration signal, and the other is the proper convergence of FS-CMA. Hence, we add recognition capability to FS-CMA to monitor the calibration signal and the convergence of the algorithm. The basic structure is shown in Figure 2.

To evaluate the performance of the FS-CMA, we define the mean square error (MSE) as

$$
\zeta=\frac{1}{N_{s}} \sum_{k=0}^{N_{s}-1}\left(\gamma-|z(k)|^{2}\right)\left(\gamma-|z(k)|^{2}\right),
$$

where $N_{s}$ is the number of samples for MSE calculation.

The implementation of recognition is based on several parameters, such as the MSE of FS-CMA, the output modulus of the subequalizer, and the signal quality of its input, the details of which will be discussed in the following section.

3.1. The Recognition of the Calibration Signal. Timing synchronization is essential for an adaptive equalizer to recover the calibration signal accurately, which is used as the training sequence. However, it is unnecessary for BE because its convergence relies on the constant modulus of the transmitted signal. Timing synchronization debugging among the multiple digital transmit/receive modules is a tough task for a DAR with thousands of channels. Hence, it is a superiority for $\mathrm{BE}$ to accomplish the convergence without rigid demand on the timing signal.

The first step of recognition is signal detection. We detect the calibration signal by comparing the modulus of the receiving signal with a predefined threshold. The envelope of the signal in the $i$ th subchannel is defined as

$$
A_{i}(k)=\sqrt{x_{I i}^{2}(k)+x_{Q i}^{2}(k)},
$$

where $x_{I i}(k)$ and $x_{\mathrm{Q} i}(k)$ denote the in-phase and quadrature component of the input signal $x_{i}(k)$, respectively.

The variance of the receiver noise $\sigma^{2}$ is recorded as a priori knowledge, and the threshold for detection is set according to the noise variance as follows:

$$
\rho_{a}=\sigma^{2}+\eta
$$


where $\eta$ is set according to the radar system. The signal is detected according to the following inequality:

$$
\begin{aligned}
A_{i}(k)<\rho_{a}, & \\
A_{i}(k+1) & >\rho_{a}, \\
\text { rising edge of the signal, } & \\
A_{i}(k) & >\rho_{a}, \\
A_{i}(k+1) & <\rho_{a},
\end{aligned}
$$

falling edge of the signal.

The pulse width and amplitude of the received calibration signal are obtained by measuring the modulus of the signal, and we will compare them with the set value getting from the control word of the radar system. We calculate the mean value of the received signal in the $i$ th subchannel as follows:

$$
\bar{A}_{i}=\frac{1}{N_{p}} \sum_{k=1}^{N_{p}} A_{i}(k),
$$

where $N_{p}$ is the number of samples above the threshold in (11).

The pulse width is obtained by counting the samples from the rising edge of the pulse to the falling edge of the pulse, which is denoted by

$$
\tau_{\mathrm{PW}}=\mathrm{TOA}_{r}-\mathrm{TOA}_{f},
$$

where $\mathrm{TOA}_{r}$ denotes the rising edge and $\mathrm{TOA}_{f}$ denotes the falling edge.

Other two important parameters are retrieved after matched filter processing (pulse compression) due to their sensitivity to the error in the receiving channel. Pulse width and amplitude of the peak after pulse compression (PC) processing are obtained. The PC processing is usually calculated as follows [16]:

$$
u(k)=\operatorname{IFFT}\left\{\operatorname{FFT}[x(k)] \cdot\{\operatorname{FFT}[p(k)]\}^{*}\right\},
$$

where FFT[ $\cdot]$ and IFFT[.] refer to fast Fourier transform (FFT) and inverse FFT, respectively.

The amplitude of the pulse after PC can be obtained by searching the maximum value of the modulus of $u(k)$

$$
A_{p}=\max \{|u(k)|\}, \quad k=1: N_{c},
$$

where $\max \{\cdot\}$ denotes searching the maximum value of the modulus and $N_{c}$ is the data length of pulse compression. We set a threshold $\rho_{p}$ to measure the peak width after PC as follows:

$$
\begin{aligned}
&|u(k)|<\rho_{p}, \\
&|u(k+1)|>\rho_{p}, \text { rising edge of the signal } \\
&|u(k)|>\rho_{p}, \\
&|u(k+1)|<\rho_{p},
\end{aligned}
$$

falling edge of the signal,
TABLE 1: Conditions of quantization decision of the parameters in RDW1.

\begin{tabular}{lcc}
\hline Parameters & Decision value & Decision value \\
\hline$A_{m}$ & $A_{m}>\eta_{a}: 1$ & $A_{m} \leq \eta_{a}: 0$ \\
$\tau_{\mathrm{PW}}$ & $\tau_{\mathrm{PW}}>\eta_{T}: 1$ & $\tau_{\mathrm{PW}} \leq \eta_{T}: 0$ \\
$A_{p}$ & $A_{p}>\eta_{p}: 1$ & $A_{p} \leq \eta_{p}: 0$ \\
$\tau_{\mathrm{PC}}$ & $\tau_{\mathrm{PC}}>\eta_{c}: 1$ & $\tau_{\mathrm{PC}} \leq \eta_{c}: 0$ \\
\hline
\end{tabular}

where $\rho_{p}$ is corresponding to $-3 \mathrm{~dB}$ sidelobe level. The width of the peak is defined as

$$
\tau_{\mathrm{PC}}=|y(k)|_{\mathrm{L} 3 \mathrm{~dB}}-|y(k)|_{\mathrm{R} 3 \mathrm{~dB}},
$$

where $|y(k)|_{\mathrm{L} 3 \mathrm{~dB}}$ refers to the left side of the $3 \mathrm{~dB}$ pulse width after $\mathrm{PC}$ and $|y(k)|_{\mathrm{R} 3 \mathrm{~dB}}$ refers to the right side of the $3 \mathrm{~dB}$ pulse width after PC.

We define a recognition description word (RDW) to evaluate the recognition results on the calibration signal, which consists of the parameters discussed above. We term the word as

$$
\mathrm{RDW} 1=\left[A_{m}, \tau_{\mathrm{PW}}, A_{p}, \tau_{\mathrm{PC}}\right]
$$

If the estimated value of the parameter is within the scope of the error tolerance, we set 1 as the parameter value; in return, we set 0 as the parameter value. The quantization decision conditions of the parameters in (19) are shown in Table 1 .

\subsection{Recognition of the Convergence of FS-CMA}

3.2.1. Surveillance of the MSE. Firstly, we define the instantaneous error of FS-CMA as

$$
e_{s}(k)=\left(\gamma-|z(k)|^{2}\right)\left(\gamma-|z(k)|^{2}\right)^{*}
$$

and a filter of $N_{h} \times 1$ dimension as

$$
\mathbf{h}=\left[\begin{array}{llll}
1 & 1 & \cdots & 1
\end{array}\right]^{T}
$$

As we all know, the MSE curve of an adaptive algorithm is achieved by Monte Carlo simulation of hundreds of times, while what we need is to surveillance the convergence property during one convergence process. Hence, we define a smooth version of the error during one convergence process as

$$
\xi_{0}(k)=\mathbf{h} \otimes e_{s}(k)
$$

Surveillance of the MSE is judged by comparing $\xi_{0}(k)$ with a threshold $\rho_{M}$. If the following inequality is established

$$
\xi_{0}(k)>\rho_{M}
$$

we say that the algorithm is in a nonsteady state. Here $\rho_{M}$ is the threshold of MSE. 
3.2.2. Surveillance of the Equalizer. Center spike initialization is usually used to initialize the weight vector of FS-CMA, which is defined as $[0,0, \ldots, 0,0,1,0,0, \ldots, 0,0]^{T}$. In other words, only the center spike of the equalizer vector is initialized as one, while all the other taps are initialized as zeros. The taps neighboring the center spike (if $N_{f}$ is an even number, the center spike will be $\left|f_{N_{f} / 2}(k)\right|$; if $N_{f}$ is an odd number, the center spike will be $\left|f_{\left(N_{f}+1\right) / 2}(k)\right|$. In this paper $\left|f_{N_{f} / 2}(k)\right|$ is used to denote the center spike for simplicity) of the weight vector are usually significant, while the taps far from it are small. Though the center spike is the most significant tap during the convergence process, its value changes little in comparison with the original 1 . However, the tap beside the center spike changes obviously during the convergence process. Therefore, we define a difference between the tap $\left|f_{N_{f} / 2-1}(k)\right|$ and its time delay version as the observation parameter, which is chosen near the center spike $\left|f_{N_{f} / 2}(k)\right|$. Another reason of choosing $\left|f_{N_{f} / 2-1}(k)\right|$ instead of the taps far from the center spike lies in that those taps are usually small and easily affected by noise.

One tap coefficient $\left|f_{N_{f} / 2-1}(k)\right|$ and its time delay version $\left|f_{N_{f} / 2-1}(k-d)\right|$ in the weight vector are under observation for the surveillance of the weight. The difference between them will become small if FS-CMA converge to a steady state. The algorithm is considered to be in steady state if the following formula is established:

$$
\begin{aligned}
f_{d}(k) & =\frac{1}{N_{p}} \sum_{k=1}^{N_{p}}\left\|f_{N_{f} / 2-1}(k)|-| f_{N_{f} / 2-1}(k-d)\right\| \\
& <\rho_{f},
\end{aligned}
$$

where $d$ denotes the time delay between the two iterations and $\rho_{f}$ is the threshold of equalizer tap difference.

Tapering is an important property of the tap weights if a proper convergence is achieved. Center spike initialization is usually used for the weight vector of $\mathrm{BE}$, and thus we compare $\left|f_{N_{f} / 2+1}(k)\right|$ with $\left|f_{0}(k)\right|$ of the $i$ th channel to express the effect of tap tapering. With the convergence of FS-CMA, the equalizer taps all converge to steady state, and the state can be denoted by the ratio between a tap near the center spike $\left|f_{N_{f} / 2+1}(k)\right|$ and a tap far from it, such as $\left|f_{0}(k)\right|$. The inequality is expressed as

$$
f_{r}(k)=\frac{\left|f_{N_{f} / 2+1}(k)\right|}{\left|f_{0}(k)\right|}>\rho_{T},
$$

where $\rho_{T}$ is the threshold of equalizer tap ratio. The establishment of (25) indicates that the equalizer is in a tapering shape.

3.2.3. Surveillance of the Equalizer Output. The amplitude of the output of the equalizer must be larger than a threshold $\rho_{0}$ according to the input calibration signal. We judge the status of the output signal by using the mean value of its modulus as follows:

$$
s_{o}=\frac{1}{N_{p}} \sum_{k=1}^{N_{p}}|z(k)|>\rho_{o} .
$$

TABLE 2: Conditions of quantization decision of the parameters in RDW2.

\begin{tabular}{lcc}
\hline Parameters & Decision value & Decision value \\
\hline$\xi_{0}(k)$ & $\xi_{0}(k) \leq \rho_{M}: 1$ & $\xi_{0}(k)>\rho_{M}: 0$ \\
$f_{d}(k)$ & $f_{d}(k)<\rho_{f}: 1$ & $f_{d}(k) \geq \rho_{f}: 0$ \\
$s_{o}$ & $s_{o}>\rho_{o}: 1$ & $s_{o} \leq \rho_{o}: 0$ \\
$f_{r}(k)$ & $f_{r}(k)>\rho_{T}: 1$ & $f_{r}(k) \leq \rho_{T}: 0$ \\
\hline
\end{tabular}

TABLE 3: Conditions of the numerical simulations.

\begin{tabular}{lcc}
\hline Simulation conditions & Simulation I & Simulation II \\
\hline Transmitted signal $p(k)$ & BPSK & BPSK \\
SNR & $45 \mathrm{~dB}$ & $45 \mathrm{~dB}$ \\
Pulse magnitude & 1 & 1 \\
Pulse width & 5000 & 2000 \\
Dimension of the equalizer $N_{f}$ & $16 \times 2=32$ & $16 \times 2=32$ \\
Oversampling rate $M$ & 2 & 2 \\
Step size & $5 \times 10^{-3}$ & $5 \times 10^{-3}$ \\
\hline
\end{tabular}

If (26) is established, we say that the output signal is qualified. Here, $\rho_{o}$ is the threshold of the modulus of the equalizer output.

According to the above discussion, we define the RDW of observing the convergence property of FS-CMA as

$$
\operatorname{RDW} 2=\left[\xi_{0}(k), f_{d}(k), s_{o}, f_{r}(k)\right] .
$$

In (27), we set 1 as the parameter value if it is within the scope of the error tolerance. On the contrary, we set 0 as the parameter value if it is larger than the error tolerance. The quantization decision conditions of the parameters in (27) are shown in Table 2.

If there is one or more than one zeros occurring in the RDW of (19) and (27), we will switch the equalizer to a status of pure time delay. It is a recognized equalizer only if all the values in the two RDWs are ones.

\section{Numerical Simulations}

We designed a series of numerical simulations to test the performance of the proposed FS-CMA with recognition capability. In simulation I, the performance of the FS-CMA with oversampling rate 2 is validated in comparison with that of CMA. In simulation II, we test the performance of the capability of recognition in various special scenarios. Some conditions for the simulations are shown in Table 3. Calibration signals in binary phased shift keying (BPSK) modulation with random codes are utilized in the following simulations.

Two oversampled channels termed as $\mathbf{c}_{a}$ and $\mathbf{c}_{b}$ are shown in Table 4, and the differences of amplitude and phase between the two channels are illustrated in Figures 3(a) and 3(b). The amplitude difference is about $2 \mathrm{~dB}$ (peak to peak), and the phase difference is about 10 degrees (peak to peak). Some values of the parameters of recognition are set as shown in Table 5. 


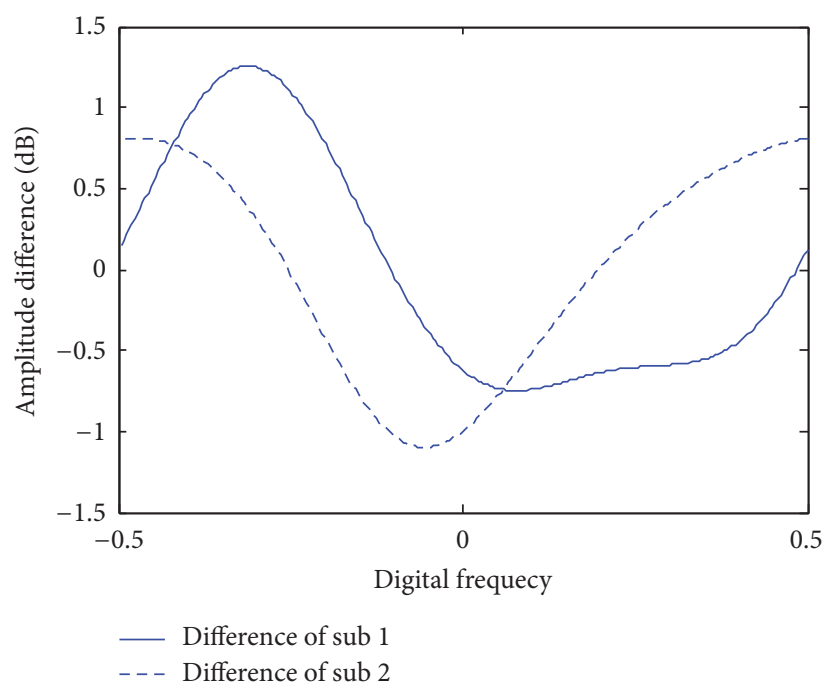

(a) Amplitude difference

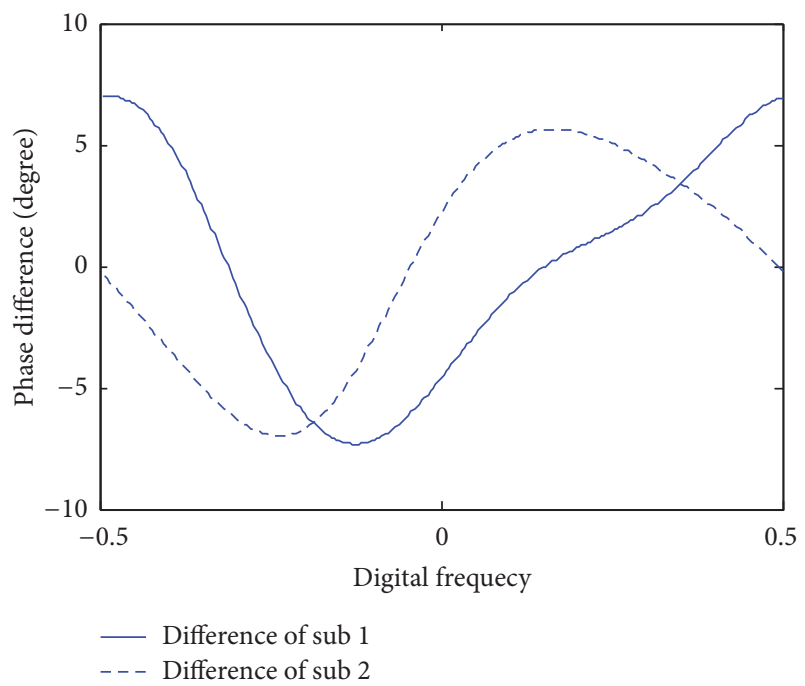

(b) Phase difference

FIgURE 3: The difference between the two channels (sub $1=$ subchannel 1, sub 2 = subchannel 2).

4.1. Simulation I. In this simulation we will show the performance difference of CMA $(M=1)$ and FS-CMA $(M=2)$ by drawing the curves of MSE and parameters of recognition, which are shown in Figure 4(a). To study the performance of the FS-CMA, Monte Carlo simulation of 500 times is carried out. The MSE curves are the mean of the errors for the 500time simulations. FS-CMA is the basic algorithm for the radar channel equalization, while the parameters recognizing the convergence property can be observed simultaneously. Figure 4(a) illustrates that a performance improvement of $2 \mathrm{~dB}$ is achieved by the FS-CMA in comparison with that of CMA. No CMA with Nyquist data rate in finite impulse response (FIR) structure can perfectly equalize a nontrivial finite impulse response channel [17]. However, FS-CMA with higher data rate can achieve perfect equalization if the subequalizer length $N_{f}$ and the subchannel length $Q$ satisfy the following requirement [17]:

$$
N_{f} \geq Q-1 .
$$

The convergence condition of FS-CMA is that the subchannels do not have any common zeros, which is easy to be satisfied in comparison with CMA. In this simulation, we choose $N_{f}=16$ and $Q=3$, which meets the condition of (28). This is the reason why the error of FS-CMA is lower than that produced by CMA in Figure 4.

With the convergence of the FS-CMA, the parameters we define to surveillance of the process also converge as shown in Figures 4(b), 4(c), and 4(d). Figure 4(b) shows the trajectories of the taps, and we can see that most of the taps are small except several significant ones. Figure 4(c) depicts the modulus of the parameter $f_{d}(k)$ in (24), which converges to steady state similar to the MSE curve in Figure 4(a). Figure 4(d) illustrates the modulus of the parameter $f_{r}(k)$ in (25). There are sharp fluctuations in Figure 4(d) because the energy of the small tap varies dramatically, which can be seen in Figure 4(b). Nevertheless, the ratios are all above certain threshold after the convergence.

We observe the channel mismatch after the equalization of FS-CMA by calculating the amplitude and phase of the output signals of the two channels. Figure 5 illustrates the differences of amplitude and phase between the two channels after equalization. The residuals of such order of magnitude after equalization will have little effect on the performance of DBF or side lobe cancellation, though small residuals in amplitude and phase can still be observed.

In this simulation, the decision values of the two RDWs are all ones; that is, RDW1 $=[1,1,1,1]$ and RDW2 $=$ $[1,1,1,1]$. It is a recognized equalizer only if all the values in the two RDWs are ones.

4.2. Simulation II. Some factors affecting the convergence of the FS-CMA are considered in this section, including the bit error in the input data and the error of the timing signal.

4.2.1. The Influence of Bit Error. Suppose there is a bit error occurring in the input data flow of the FS-CMA, we get the convergence process of the RDW as shown in Figure 6. The simulated bit error is added in the 4500th point of the input data.

Figure 6 shows that the FS-CMA diverges at the 4500th point and restarts the convergence process for the second time. However, the convergence process is still in an unsteady state at the end of the received signal. The value of the RDW2 achieved here is RDW2 $=[0,0,1,1]$. The equalizer attained under this situation is not qualified because that there are two zeros in RDW2. We switch the equalizer to a pure time delay vector, which is vector of zeros with only one spike in the position of the 8th coefficient.

4.2.2. Influence of the Error of Timing Signal. If there are some problems occurring on the timing signal, only a section of 


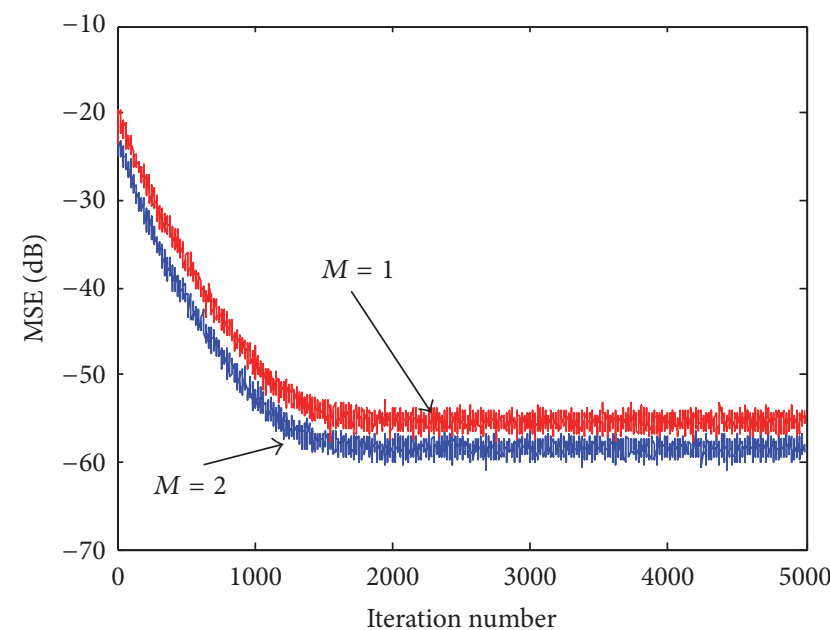

(a) The performance of FSE with different oversampling rate

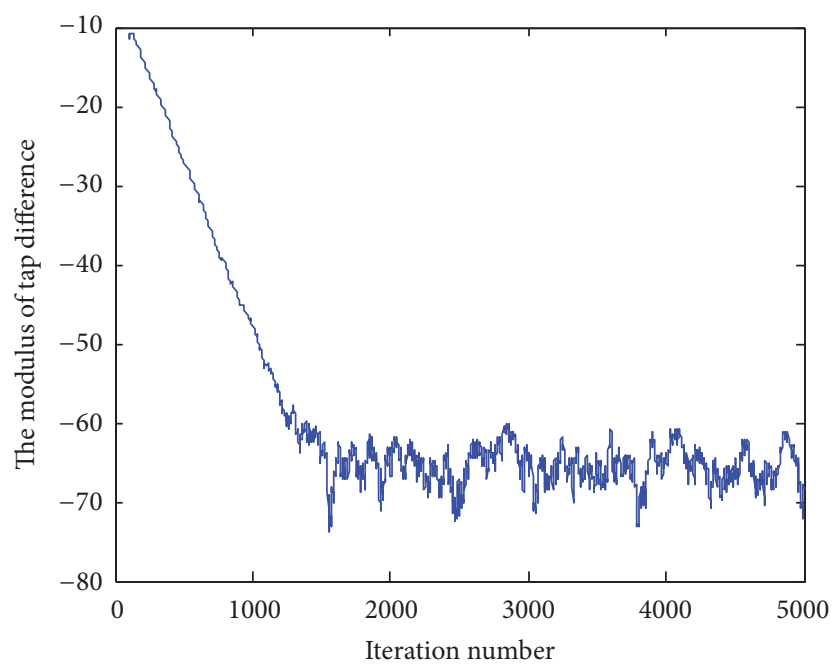

(c) The convergence of the difference of the tap

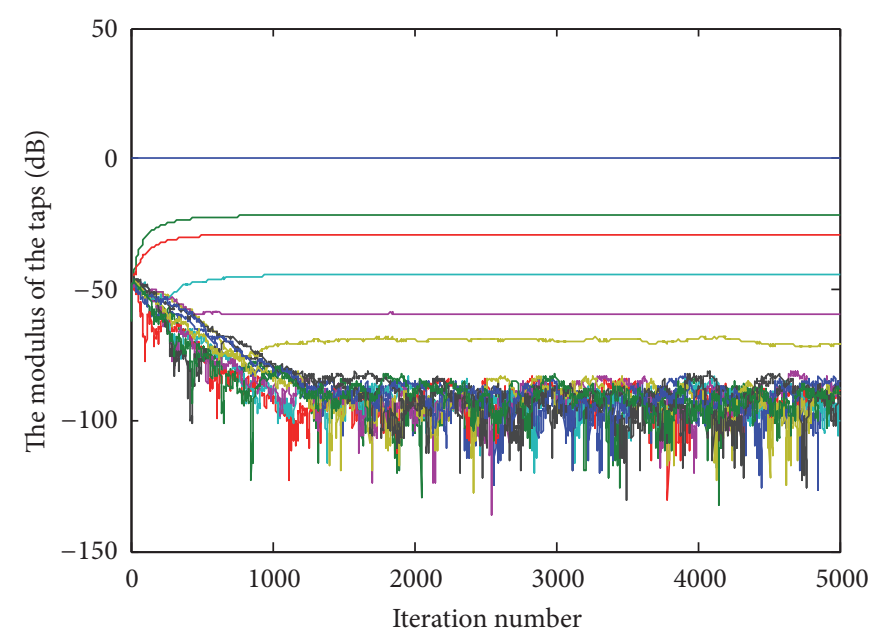

(b) The trajectories of the taps

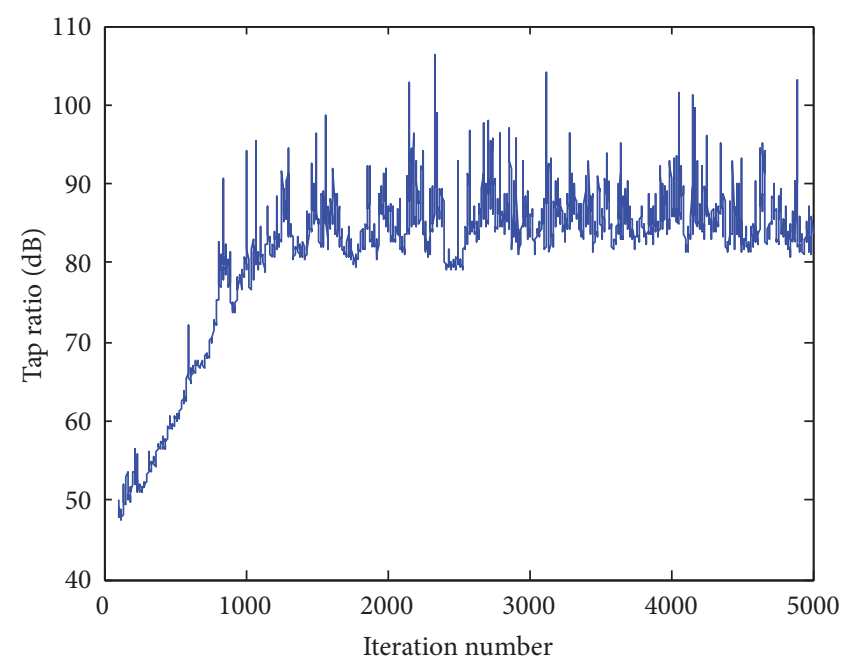

(d) The convergence of the ratio of the taps

FIGURE 4: The convergence property of FS-CMA and the recognition parameters.

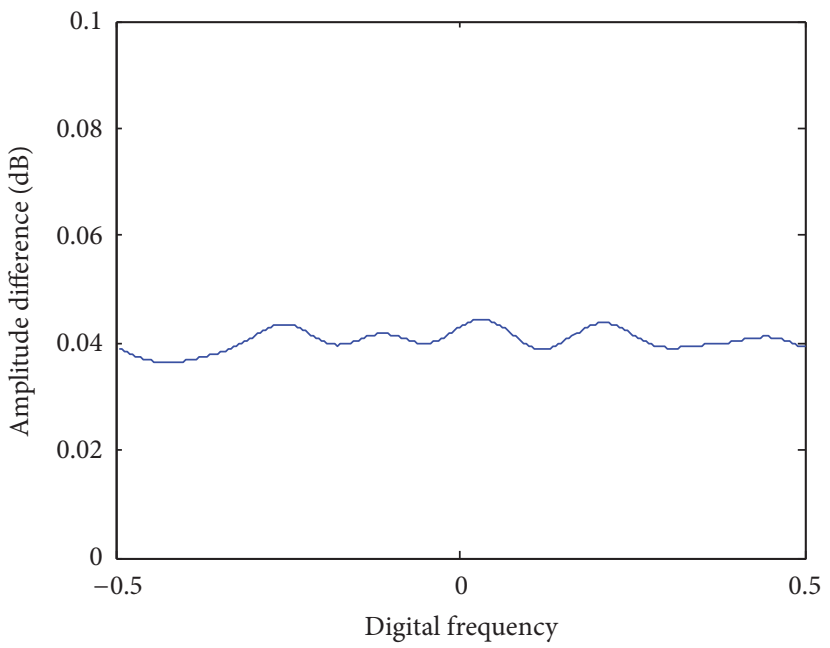

(a) Amplitude difference

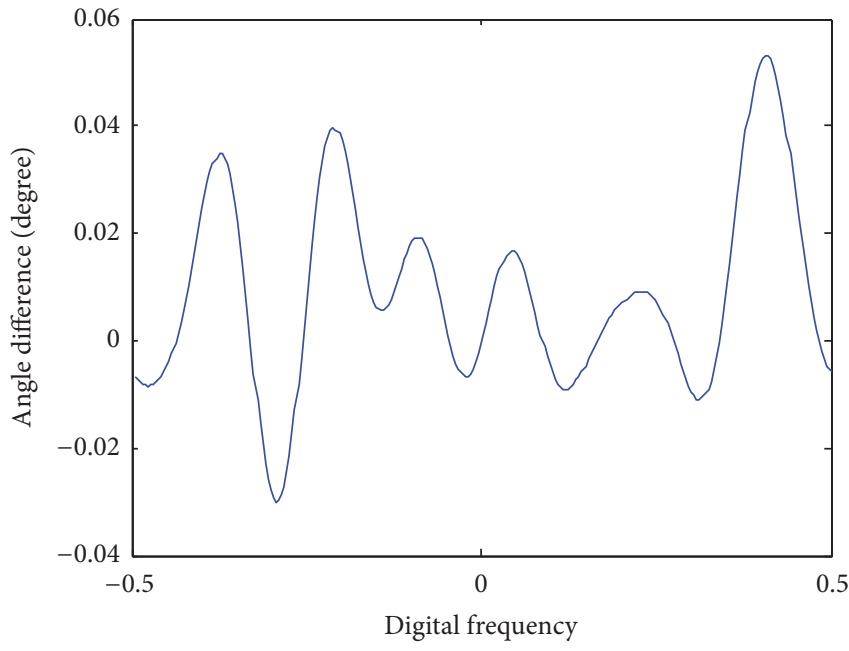

(b) Phase difference

FIGURE 5: The difference of the frequency responses after equalization. 
TABLE 4: Coefficients of the two channels.

\begin{tabular}{ccccc}
\hline Channel & Subchannels & Ray 1 & Ray 2 & Ray 3 \\
\hline \multirow{2}{*}{1} & Sub 1 $c_{a 1}$ & $c_{a 1}^{0}=1$ & $c_{a 1}^{1}=0.08 e^{j 0.4}$ & $c_{a 1}^{2}=-0.04 e^{j 1.4}$ \\
& Sub 2 $\mathbf{c}_{a 2}$ & $c_{a 2}^{0}=1$ & $c_{a 2}^{1}=-0.05 e^{j 1.7}$ & $c_{a 2}^{2}=-0.02 e^{-j 3.2}$ \\
2 & Sub 1 $c_{b 1}$ & $c_{b 1}^{0}=1$ & $c_{b 1}^{1}=0.06 e^{j 2.1}$ & $c_{b 1}^{2}=-0.023 e^{j 0.9}$ \\
& Sub 2 $c_{b 2}$ & $c_{b 2}^{0}=1$ & $c_{b 2}^{1}=-0.072 e^{-j 2.4}$ & $c_{b 2}^{2}=-0.02 e^{-j 1.1}$ \\
\hline
\end{tabular}

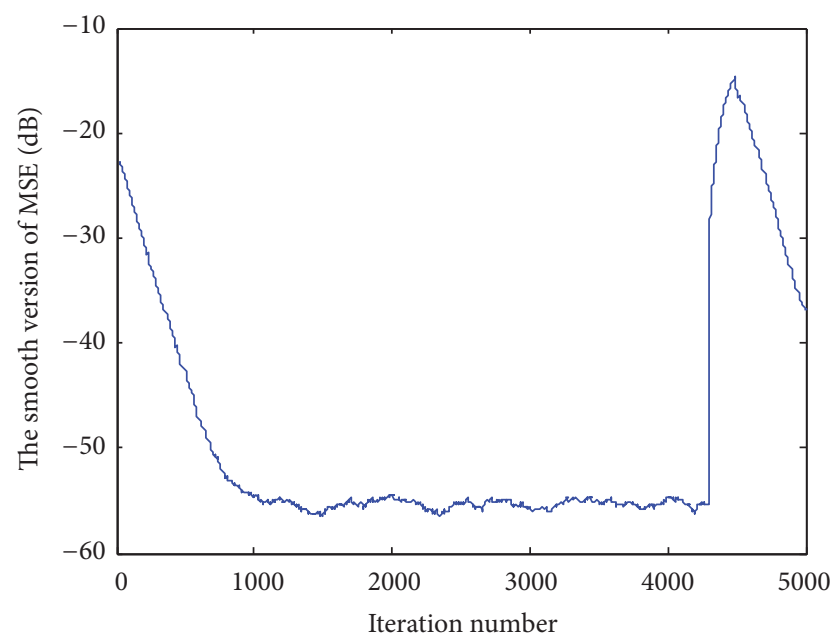

(a) The smooth version of MSE

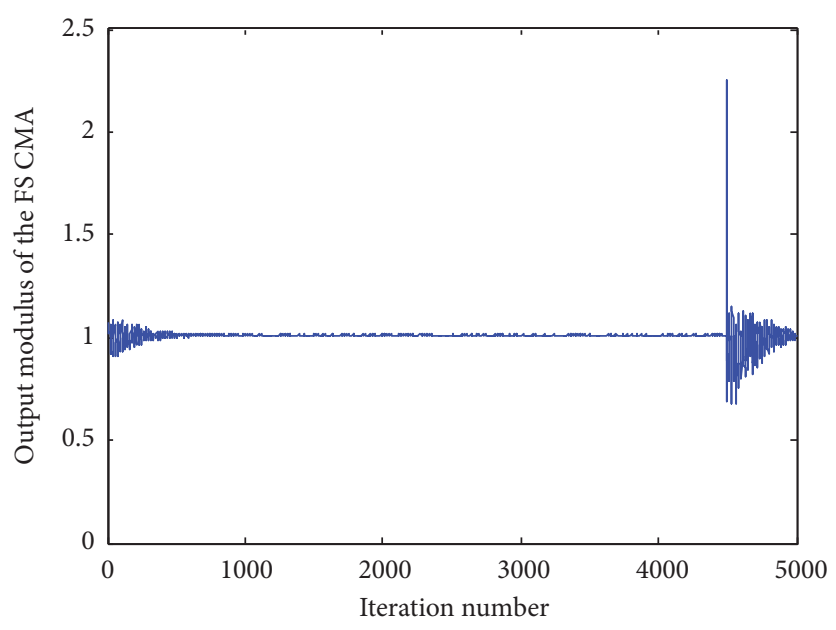

(c) The modulus of the FS-CMA output

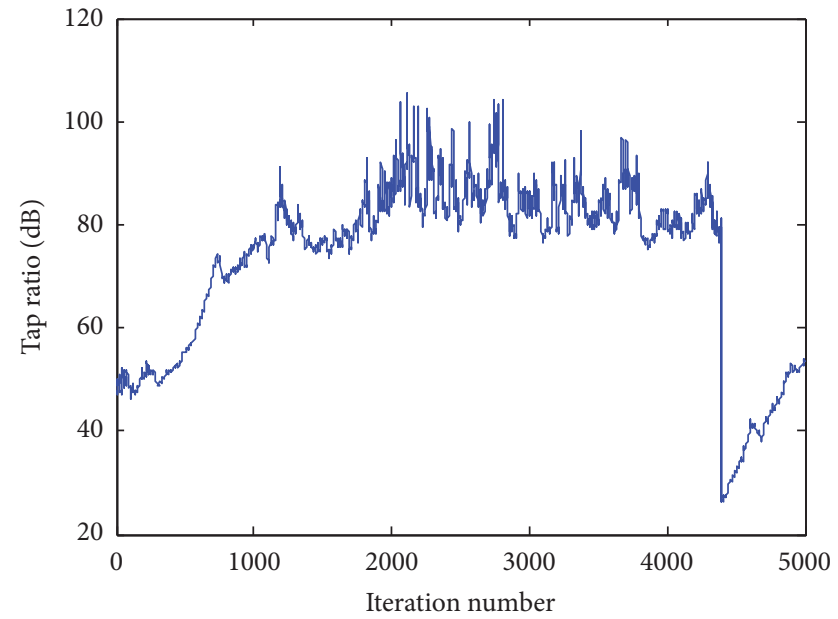

(b) The difference between $\left|f_{7}(k)\right|$ and $\left|f_{7}(k-100)\right|$

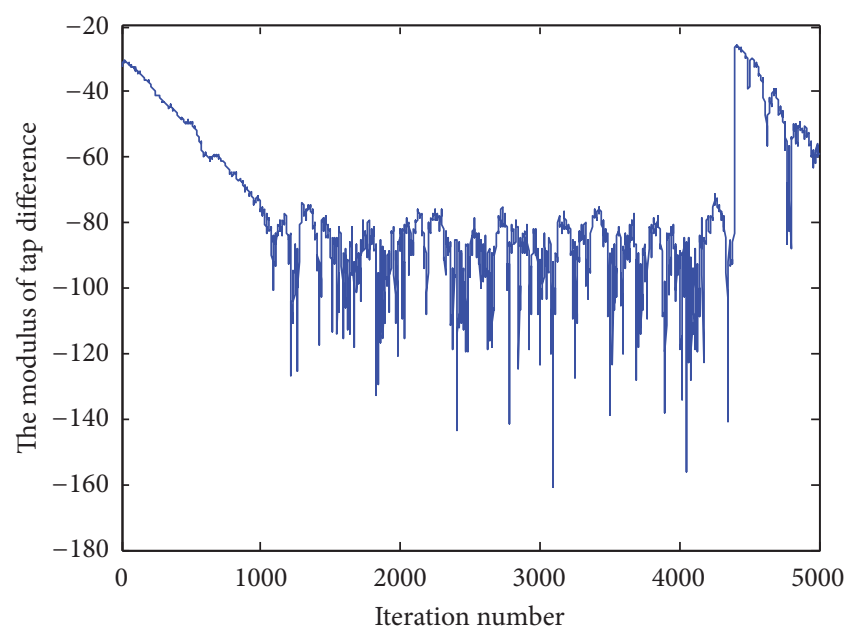

(d) The ratio of $\left|f_{7}(k)\right| /\left|f_{0}(k)\right|$

FIGURE 6: The changes of the parameters in the RDW.

the calibration signal has been sampled for equalization. Figure 7(a) illustrates that only 2000 samples are obtained in this simulated calibration process. More than half of the signal is missing due to the error of the timing signal for calibration sampling. The peak width after PC is in inverse ratio to the signal bandwidth, as shown in Figure 7(b). From Figure 7(b) we can see that the peak width remains unchanged because the signal bandwidth remains unchanged. Hence, the bandwidth of the system is still covered by the calibration signal. It is a merit for the use of stochastic phase modulation signal.
The parameters in the RDW are shown in Figure 8, and the decision values of the two RDWs are all ones; that is, RDW1 $=[1,1,1,1]$ and RDW2 $=[1,1,1,1]$. We can conclude from Figure 8 that 2000 samples will be enough for the convergence of the FS-CMA. More sample inputs will provide high probability of robustness to the variation of the timing signal. The convergence of the equalizer does not depend on the exact signal, which is another merit of the BE. In other words, we can say that $\mathrm{BE}$ will be more stable against the timing signal. 


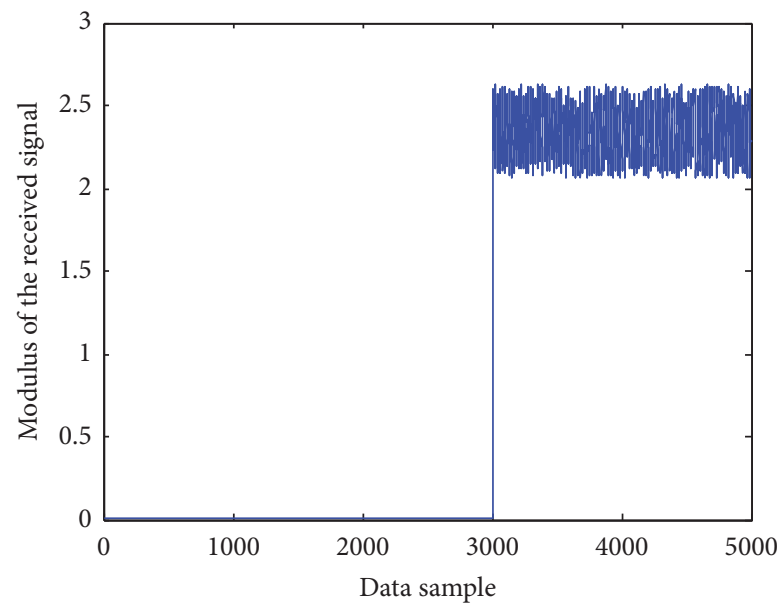

(a) The modulus of the received signal

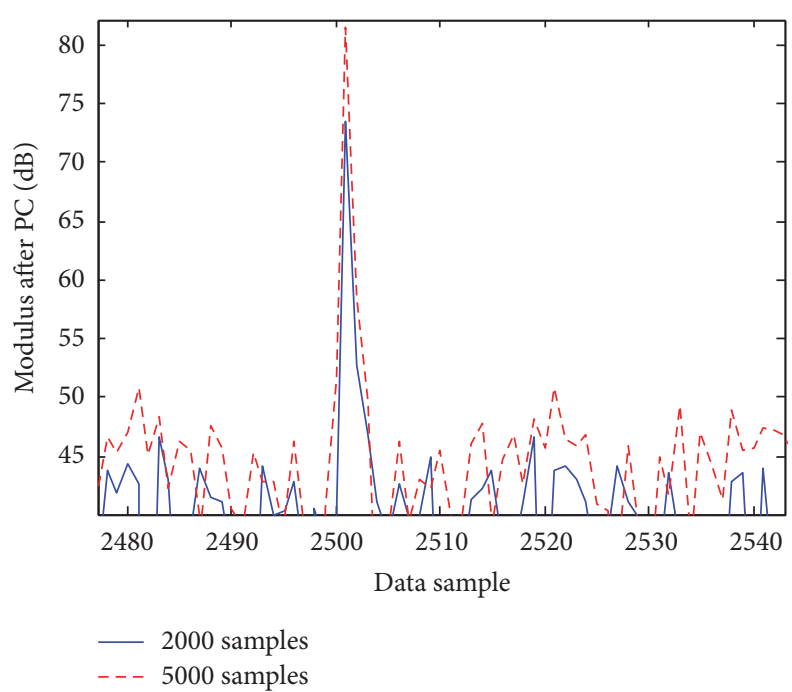

(b) The modulus after PC

FIgURE 7: The received calibration signal.

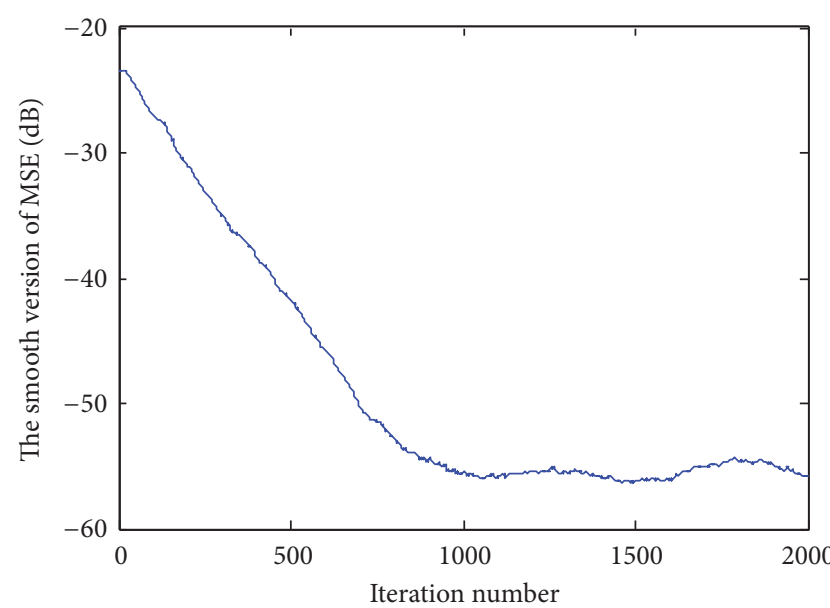

(a) The smooth version of MSE

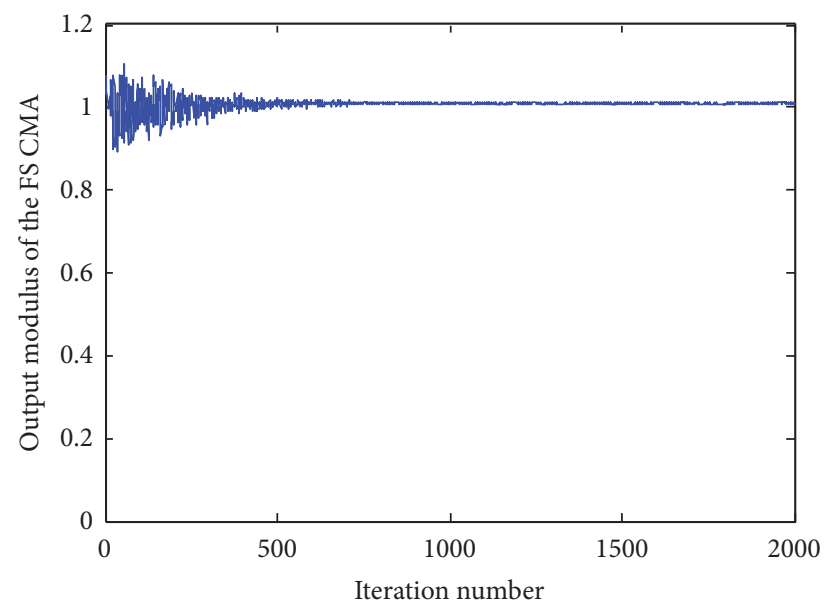

(c) The modulus of the FS-CMA output

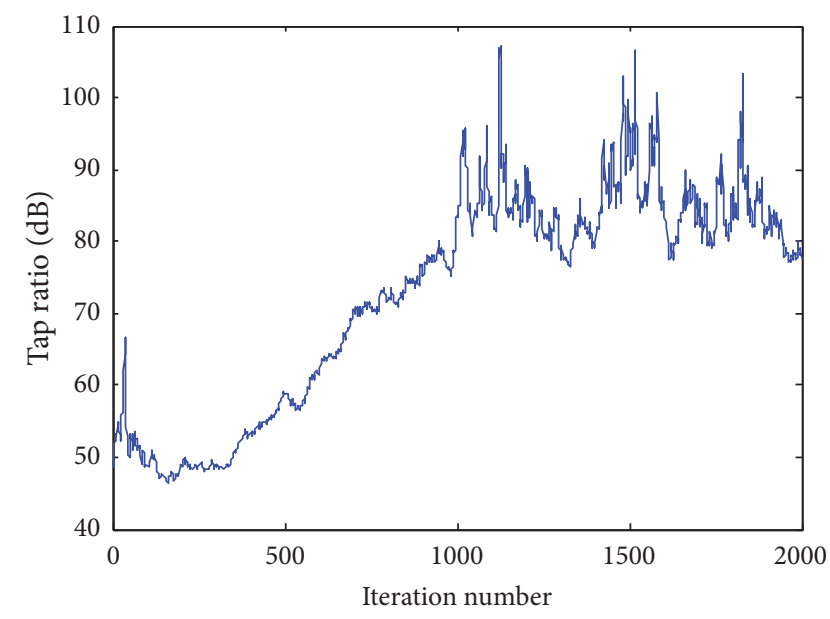

(b) The ratio of $\left|f_{7}(k)\right| /\left|f_{0}(k)\right|$

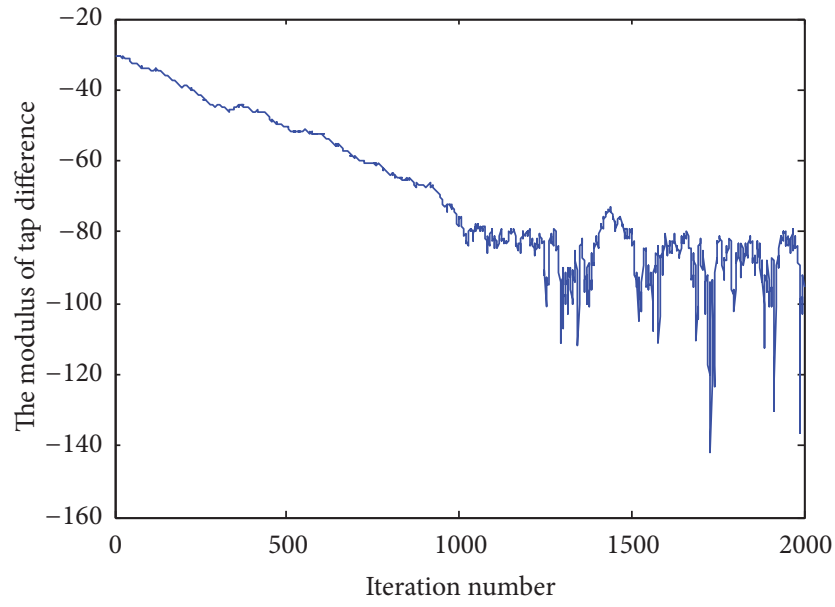

(d) The difference between $\left|f_{7}(k)\right|$ and $\left|f_{7}(k-100)\right|$

FIGURE 8: The changes of the parameters in the RDW. 
TABLE 5: Parameters of recognition.

\begin{tabular}{lcc}
\hline Parameters & Set values & Unit \\
\hline$\rho_{T}$ & 50 & $\mathrm{~dB}$ \\
$\rho_{o}$ & 0.5 & Number \\
$\rho_{M}$ & -50 & $\mathrm{~dB}$ \\
$\rho_{f}$ & -60 & $\mathrm{~dB}$ \\
$\eta_{a}$ & 0.5 & Number \\
$\eta_{T}$ & 1500 & Samples \\
$\eta_{p}$ & 20 & $\mathrm{~dB}$ \\
$\eta_{c}$ & 2 & Sample interval \\
\hline
\end{tabular}

\section{Conclusions}

Blind equalization with recognition capability is investigated for channel equalization of a DAR. The recognition capability aims at improving the equalization performance in various situations. Different scenarios such as the error of the timing signal and divergence of the $\mathrm{BE}$ have been emulated to evaluate the performance of recognition, which is validated via numerical simulations.

\section{Competing Interests}

The authors declare that they have no competing interests.

\section{Acknowledgments}

This work was partially supported by the Natural Science Foundation of Jiangsu Province (Grants nos. BK20151501 and BK20140858), Fundamental Research Funds for the Central Universities (Grant no. 2015B03014), and the National Natural Science Foundation of China (Grant no. 61401145).

\section{References}

[1] S. H. Talisa, K. W. O’Haver, T. M. Comberiate, M. D. Sharp, and O. F. Somerlock, "Benefits of digital phased array radars," Proceedings of the IEEE, vol. 104, no. 3, pp. 530-543, 2016.

[2] D. J. Rabideau, R. J. Galejs, F. G. Willwerth, and D. S. McQueen, "An S-band digital array radar testbed," in Proceedings of the 6th IEEE Phased Array Systems and Technology Symposium (Array '03), pp. 113-118, Boston, Mass, USA, October 2003.

[3] E. Makhoul, A. Broquetas, F. López-Dekker, J. Closa, and P. Saameno, "Evaluation of the internal calibration methodologies for spaceborne synthetic aperture radars with active phased array antennas," IEEE Journal of Selected Topics in Applied Earth Observations and Remote Sensing, vol. 5, no. 3, pp. 909-918, 2012.

[4] W. X. Li, J. Z. Lin, Y. Zhang, and Z. Chen, "FIR-filter-based method for the calibration of model errors in wideband digital array radar," Electronics Letters, vol. 52, no. 10, pp. 867-868, 2016.

[5] D. N. Godard, "Self-recovering equalization and carrier tracking in two-dimensional data communication systems," IEEE Transactions on Communications, vol. 28, no. 11, pp. 1867-1875, 1980.

[6] C. R. Johnson Jr., P. Schniter, T. J. Endres, J. D. Behm, D. R. Brown, and R. A. Casas, "Blind equalization using the constant modulus criterion: a review," Proceedings of the IEEE, vol. 86, no. 10, pp. 1927-1949, 1998.

[7] Y. Li and Z. Ding, "Global convergence of fractionally spaced godard (CMA) adaptive equalizers," IEEE Transactions on Signal Processing, vol. 44, no. 4, pp. 818-826, 1996.

[8] G. B. Giannakis and S. D. Halford, "Blind fractionally spaced equalization of noisy FIR channels: direct and adaptive solutions," IEEE Transactions on Signal Processing, vol. 45, no. 9, pp. 2277-2292, 1997.

[9] S. Haykin, Y. Xue, and P. Setoodeh, "Cognitive radar: step toward bridging the gap between neuroscience and engineering," Proceedings of the IEEE, vol. 100, no. 11, pp. 3102-3130, 2012.

[10] K. L. Bell, C. J. Baker, G. E. Smith, J. T. Johnson, and M. Rangaswamy, "Cognitive radar framework for target detection and tracking," IEEE Journal on Selected Topics in Signal Processing, vol. 9, no. 8, pp. 1427-1439, 2015.

[11] S. Lu, W. Yi, G. Cui, L. Kong, and X. Yang, "Design and application of dynamic environmental knowledge base," IET Radar, Sonar \& Navigation, vol. 10, no. 6, pp. 1118-1126, 2016.

[12] U. Güntürkün, "Toward the development of radar scene analyzer for cognitive radar," IEEE Journal of Oceanic Engineering, vol. 35, no. 2, pp. 303-313, 2010.

[13] M. Rasekh and S. R. Seydnejad, "Design of an adaptive wideband beamforming algorithm for conformal arrays," IEEE Communications Letters, vol. 18, no. 11, pp. 1955-1958, 2014.

[14] Y.-H. Chen and C.-H. Chen, "A new structure for adaptive broadband beamforming," IEEE Transactions on Antennas and Propagation, vol. 39, no. 4, pp. 551-555, 1991.

[15] S.-C. Lin and C.-W. Wu, "Spatial-temporal fractionally spaced decision-feedback equalisation for fading channels with dispersive interference," IET Communications, vol. 5, no. 11, pp. 15501559, 2011.

[16] K. Brophy and N. Curnow, Radar, Walleah Press, 2012.

[17] C. B. Papadias and D. T. M. Slock, "Fractionally spaced equalization of linear polyphase channels and related blind techniques based on multichannel linear prediction," IEEE Transactions on Signal Processing, vol. 47, no. 3, pp. 641-654, 1999. 


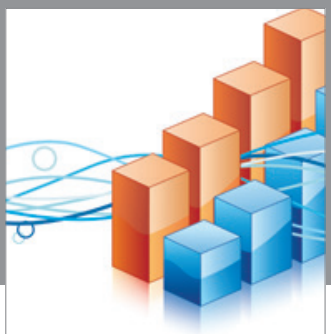

Advances in

Operations Research

vatem alat4

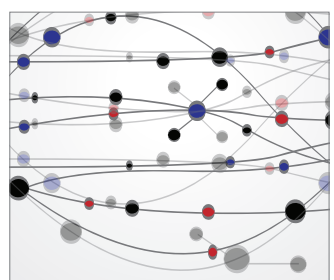

\section{The Scientific} World Journal
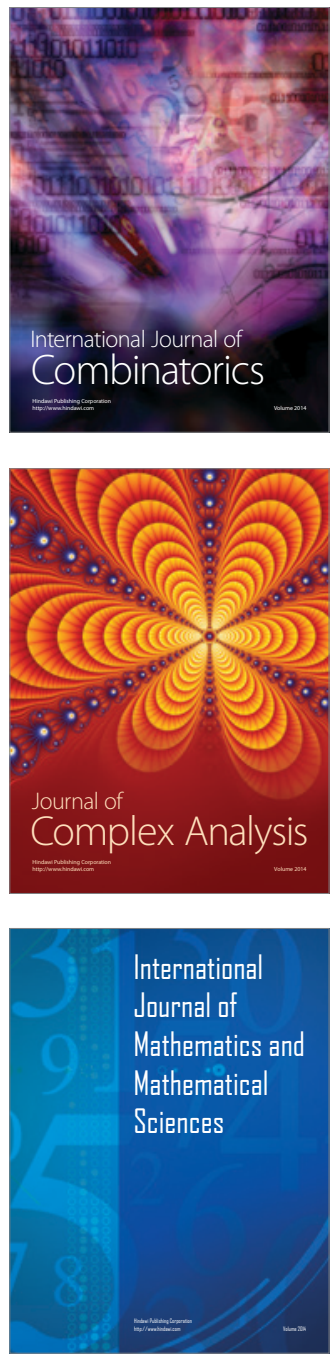
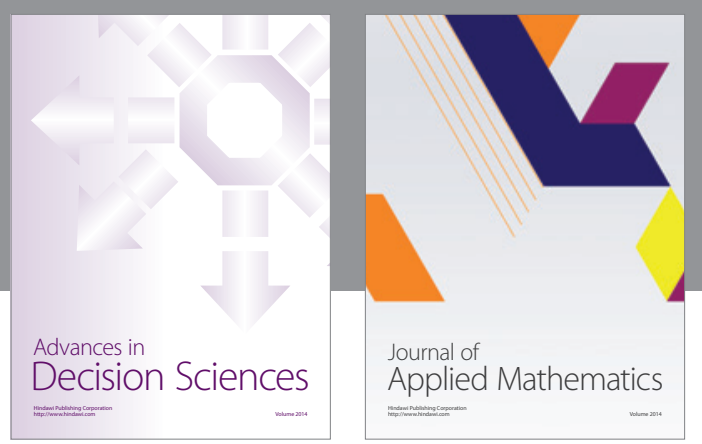

Algebra

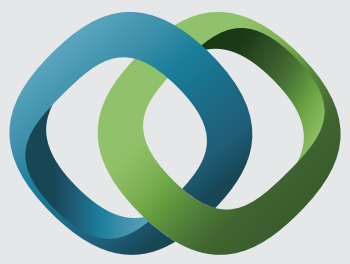

\section{Hindawi}

Submit your manuscripts at

https://www.hindawi.com
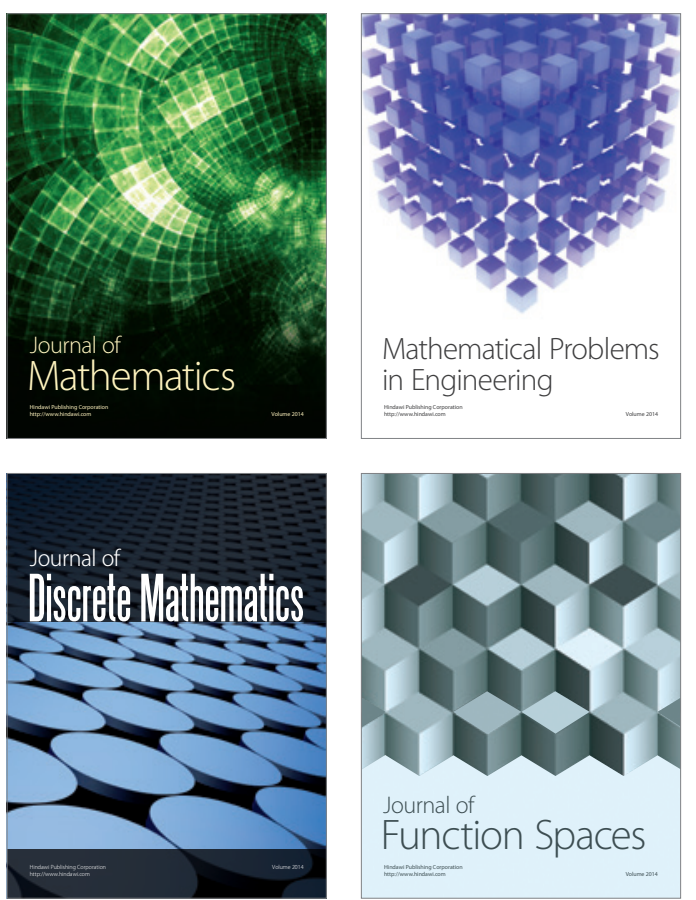

Mathematical Problems in Engineering
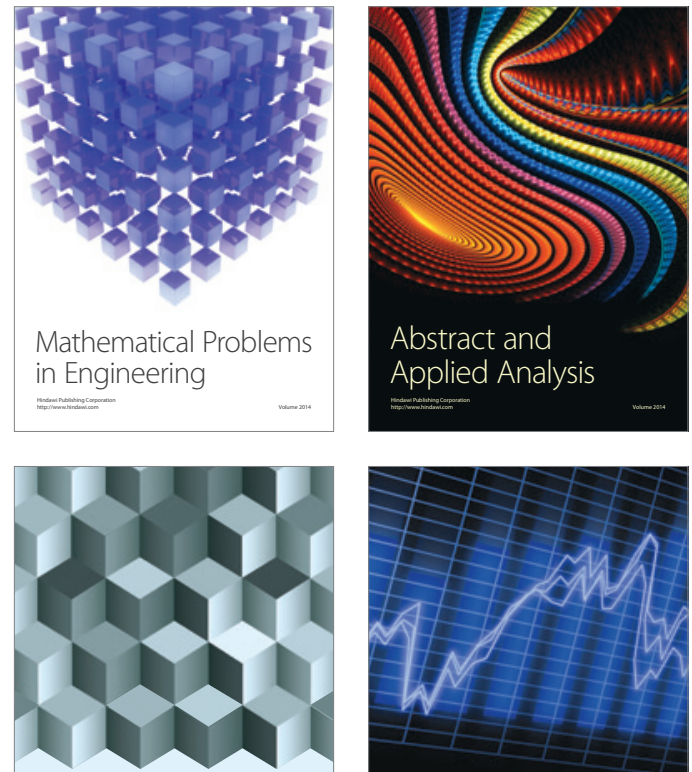

Journal of

Function Spaces

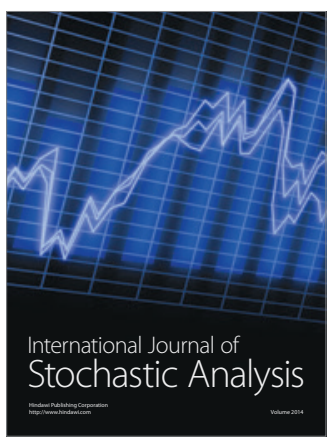

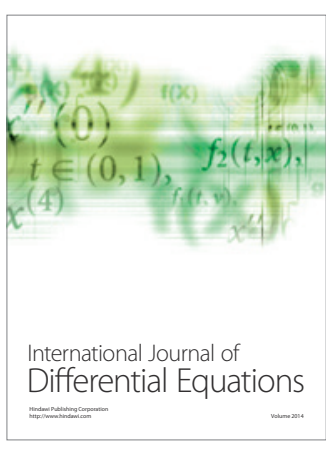
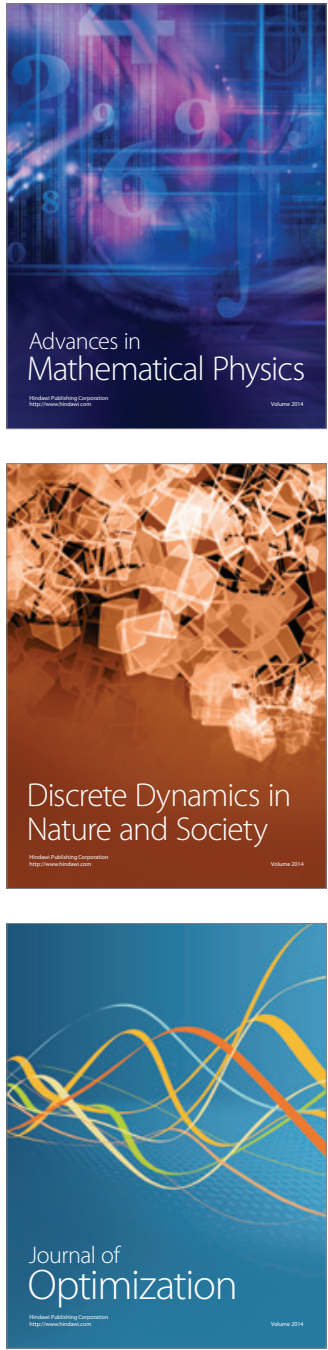